\title{
Precision Astrometry with Adaptive Optics: Constraints on the Mutual Orbit of Luhman 16AB from GeMS
}

\author{
S. Mark Ammons ${ }^{\mathrm{a}}$, E. Victor Garcia ${ }^{\mathrm{a}}$, Maissa Salama ${ }^{\mathrm{c}}$, Benoit Neichel ${ }^{\mathrm{b}, \mathrm{k}}$, Jessica Lu ${ }^{\mathrm{d}}$, Christian \\ Marois $^{\mathrm{e}}$, Bruce Macintosh ${ }^{\mathrm{f}}$, Dmitry Savransky $^{\mathrm{g}}$, Eduardo Bendek ${ }^{\mathrm{i}}$, Olivier Guyon ${ }^{\mathrm{j}}$, Eduardo Marin ${ }^{\mathrm{k}}$, \\ Vincent Garrel $^{\mathrm{k}}$, Gaetano Sivo ${ }^{\mathrm{k}}$ \\ ${ }^{a}$ Lawrence Livermore National Laboratory, 7000 East Ave, Livermore, CA, USA, \\ ammons1@1lnl.gov; \\ bAix Marseille University, CNRS, LAM, UMR 7326, 13388, Marseille, France; \\ ${ }^{\mathrm{c}}$ Institute for Astronomy, University of Hawaii, Honolulu, HI, USA; \\ ${ }^{\mathrm{d}}$ University of California, Berkeley;

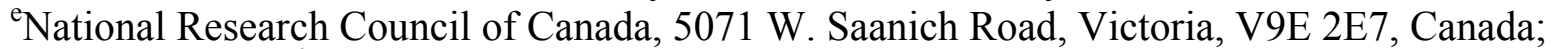 \\ ${ }^{\mathrm{f}}$ Stanford University, 450 Serra Mall, Stanford, CA, USA; \\ ${ }^{\mathrm{g}}$ Cornell University, Ithaca, NY, USA; \\ ${ }^{i}$ NASA Ames Research Center, Moffett Field, CA, USA; \\ ${ }^{\mathrm{j}}$ University of Arizona, Tucson, AZ, 85721, USA; \\ ${ }^{\mathrm{k}}$ Gemini Observatory, La Serena, Chile
}

\begin{abstract}
ELTs equipped with MCAO systems will be powerful astrometric tools in the next two decades. With sparsefield precisions exceeding 30 uas for $\mathrm{V}>18$, the ELTs will surpass even GAIA's per-epoch precision for faint stars $(\mathrm{V}>12)$. We present results from an ongoing astrometry program with Gemini GeMS and discuss synergies with WFIRST and GAIA. First, we present a fit to the relative orbit of the individual L/T components of Luhman $16 \mathrm{AB}$, the nearest brown dwarf binary known. Exploiting GeMS' wide field of view to image reference stars, we are able to track the relative motion to better than 0.2 mas. We find that a mutual Keplerian orbit with no perturbing planets fits the binary separation to within the measurement errors, ruling out companions down to 14 earth masses for certain orbits and periods.
\end{abstract}

Keywords: astrometry, adaptive optics, multi-conjugate, tomography, M92, NGC 1851, brown dwarf

\section{INTRODUCTION}

Direct imaging and spectroscopy of exoplanets has flourished in the past decade, with more than 10 discovered through this method. ${ }^{1-5}$ Masses for such objects are typically estimated by measuring the age of the parent star separately and using theoretical models of planetary luminosity evolution to look up a best-fit mass that matches the observed luminosity of the planet and stellar age. Ideally, as the controlling parameter discriminating brown dwarfs from true planets, companion mass would be measured independently. In this way, direct imaging discoveries would be driving the development of theoretical planet formation models.

Independent mass measurements of directly imaged exoplanets have proven elusive to date. Astrometric measurement of stellar acceleration could directly constrain masses of planets in long period orbits. Astrometry and direct imaging combine to yield full orbit parameters and exoplanet masses with significant overlap between samples ${ }^{6,7}$ (see Figure 1). As a technique for constraining

Adaptive Optics Systems V, edited by Enrico Marchetti, Laird M. Close, Jean-Pierre Véran, Proc. of SPIE Vol. 9909, 99095T (C) 2016 SPIE - CCC code: 0277-786X/16/\$18 - doi: 10.1117/12.2233775 
exoplanet mass, astrometry is complementary to well-developed radial velocity techniques, which are more sensitive to smaller star-planet separations. Astrometric follow-up also comparables favorably to RV in a signal-to-noise sense: Many direct imaging surveys specifically target young stars to yield self-luminous Jovian planets, but young stars tend to have active chromospheres and thus considerable radial velocity jitter (Figure 2). In comparison, the astrometric jitter due to starspots is a smaller fraction of the signal induced by the Jovian planets detectable by direct imaging - less than 1 microarcsecond for solar type stars. ${ }^{8}$

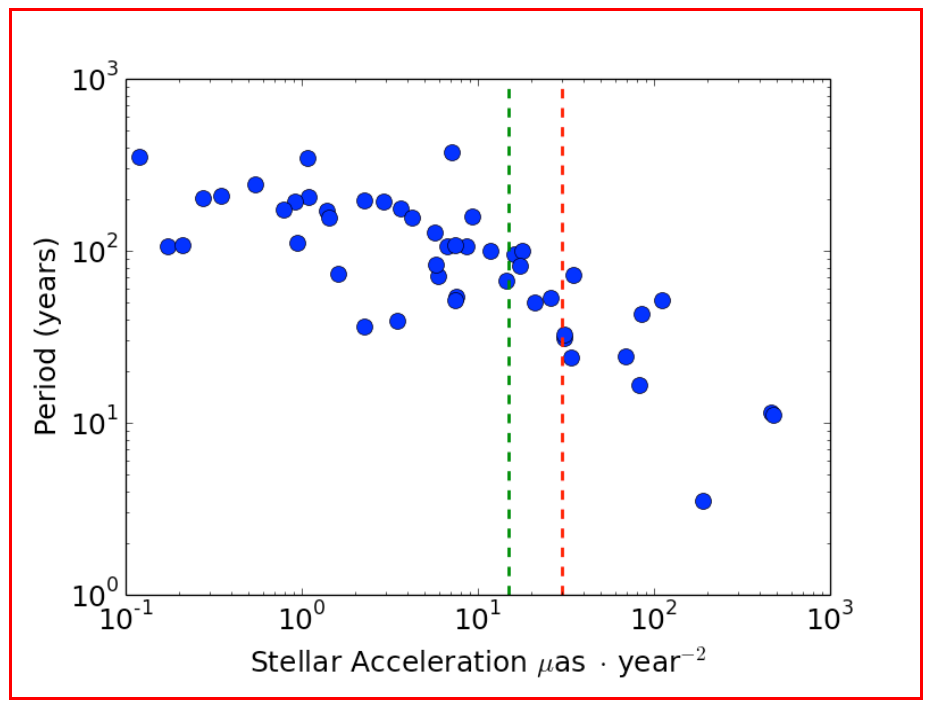

Figure 1. Measurement of stellar acceleration induced by an orbiting exoplanet can yield planet mass if the planet orbital parameters are determined from direct imaging. Such measurements will be possible for a subset of the longperiod planets discovered by high contrast direct imaging surveys, as shown above for a set of simulated planets that could be detected by next generation direct imagers.
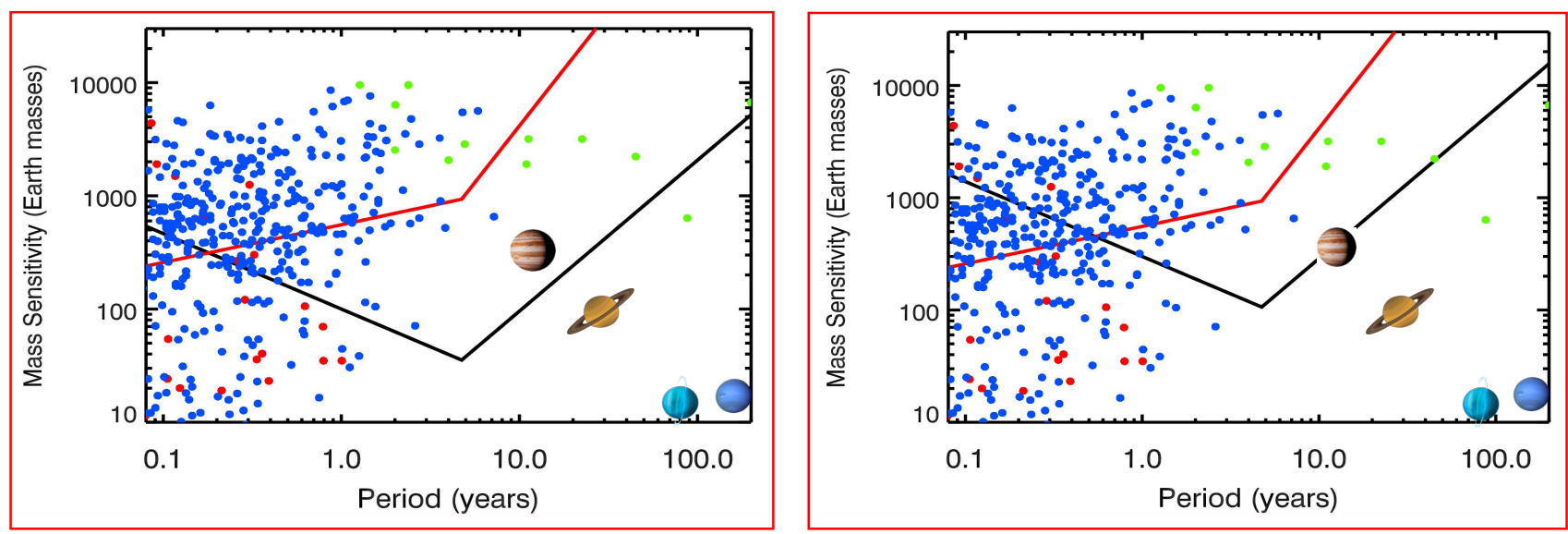

Figure 2. Mass sensitivities for radial velocity follow-up (red curves) and astrometry follow up over 5 years (black curves, $\sim 0.2$ mas precision). The left plot shows mass sensitivities for target distances of $10 \mathrm{pc}$ (left) and $30 \mathrm{pc}$ (right). Due to high stellar noise in the young stars targeted by direct imaging surveys $(\sim 50 \mathrm{~m} / \mathrm{s})$, astrometry can reach lower masses than radial velocity monitoring.

Astrometry also yields two projected components of the induced stellar acceleration vector, as opposed to one component for RV. This vector points in the direction of the planet in the case of 
single planet systems, which can be used as a prior in direct imaging data to increase the effective signal to noise of the detection. In the case of multiple planet systems, astrometry sets a constraint on a nonlinear sum of the multiple planets (depending on the orientation of the system during the astrometric follow up monitoring) and may point to additional unseen planets.

In this paper, we characterize the astrometric precision of the GeMS MCAO system, a promising instrument for exoplanet mass measurement, by constraining the relative orbit of the Luhman $16 \mathrm{AB}$ system.

\section{LUHMAN 16 RELATIVE ORBIT}

We obtained high precision astrometry of the Luhman 16 AB binary brown dwarf system using the Gemini South MCAO system and a photographic plate taken with the 1-meter Schmidt telescope at ESO's La Silla Observatory in 1984. The data span 32 years, roughly the period previously estimated for this binary. ${ }^{9,10}$ Luhman $16 \mathrm{AB}$ is the closest known L/T transition binary brown dwarf system at 2 parsecs and an ideal test case for precise relative astrometric monitoring with MCAO systems. The relative orbital period is of order 20-60 years and the stars have been characterized as L and late T brown dwarfs. ${ }^{11}$ A GeMS K-band image of Luhman16 AB is shown in Figure 3.

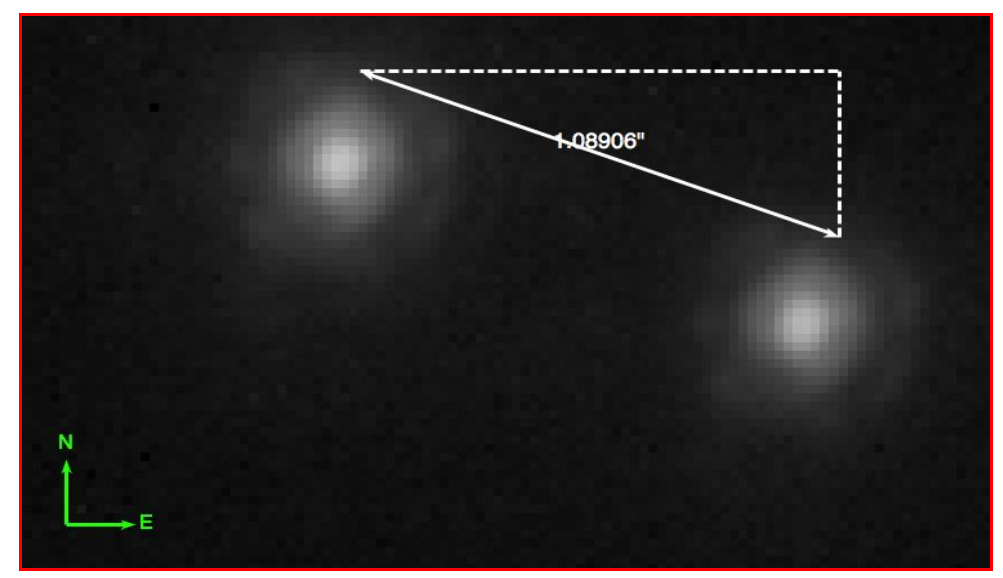

Figure 3. GeMS K-band image of Luhman 16AB binary brown dwarf system, log scale.

\subsection{GeMS MCAO Imaging}

The observations of Luhman $16 \mathrm{AB}$ and NGC1851 were obtained with the Gemini South telescope using the Gemini South Adaptive Optics Imager. ${ }^{12,13}$ Together, the two instruments deliver near diffraction limited images from 0.9-2.4 microns. GeMS is a facility AO system for the Gemini South telescope and GSAOI is a near-infrared AO camera used with GeMS. The GSAOI detector is formed by 2x2 mosaic of Rockwell HAWAII-2RG 2048x2048 pixel arrays. At the f/32 GeMS output focus, GSAOI provides 0.02 " per pixel sampling and gaps of about 3 " between arrays.

The GeMS static distortion map is measured with observations of globular cluster NGC 1851 obtained as part of the GeMS Science Verification program. Stellar positions from GeMS are compared with known stellar positions measured with the Hubble Space Telescope Advanced Camera for Surveys. ${ }^{14}$ The distortion measurement uses two sets of undithered frames of NGC 1851 
taken one month apart, on Dec 30, 2012 (set 1) and Jan 28, 2013 (set 2). Images are flat-fielded with combined twilight flats, and masked for bad pixels using IRAF's GAREDUCE reduction routines. Images are not mosaicted into 4196x4196 frames.

GeMS images of Luhman 16 AB were obtained with GeMS on the nights on February 12, April 12, April 13, May 27, December 3, 2014 and March 5, 2015. The J-continuum narrow-band filter was used to prevent saturating Luhman $16 \mathrm{AB}$. The images were taken with exposure times of 15 seconds and an instrument position angle of 200 degrees, placing Luhman 16 AB on chip \#2. AOcorrected Strehl ratios as measured on Luhman $16 \mathrm{AB}$ varied between 0.5 and $5 \%$. The data were reduced following the standard procedures for near-infrared imaging provided by the Gemini/GSAOI package inside IRAF. ${ }^{15}$ Images are flat-fielded with combined twilight flats and masked for bad pixels using IRAF's GAREDUCE reduction routines.

\subsection{GeMS Distortion Map Measurement with NGC1851 Observations}

Starfinder ${ }^{16}$ is run on individual frames, using 20 bright, unsaturated stars distributed throughout the field to create a master PSF for each frame. A first extraction is performed down to a 20-sigma limit, secondary stars in the PSF images are subtracted, and a new master PSF free from secondary sources is created. Stars are then re-extracted down to 7-sigma with a PSF correlation threshold of 0.7. The Starfinder function MATCH_COORD is used to approximately register each frame to the first in each set. Frames with fewer than 1500 stars matching the reference frame's list to within 3 pixels are rejected. This step trims Set 1 from 18 to 13 frames and Set 2 from 26 to 24 frames.

To establish a set of common stars in all frames, stars falling more than 3 pixels from the reference frame's position in any of the frames are rejected from the list, leaving 1000 stars in each set in the full GSAOI field. Stars located within 10 pixels of any other common stars are also eliminated. Stars within 2 pixels of a bad pixel or within 10 pixels of the edge of the field of view are rejected. If Starfinder splits a single star into multiple detections in single frame, those measurements are excluded (but other frames with a single detection are retained for that star). Following these exclusions, $\sim 1200$ stars remain for each set.

For each frame, a 4-parameter linear transformation is performed to transform each GeMS chip to the appropriate area on the ACS frame to enable matching between GeMS stars and ACS stars. The deviations between the ACS and original untransformed GeMS positions are measured and recorded for each star in each frame (a total of 200,000 stellar measurements). A global 120-parameter fourth-order static polynomial distortion pattern (30 per chip) in addition to a six-parameter linear transformation for each frame is fitted to all stellar deviation measurements using the IDL MPFITFUN minimization routine. The nonlinear components of the model remain constant to within 2 milliarseconds across the full field when the position angle of the instrument is rotated and the dither position is changed by up to 30 ".

The resulting nonlinear components of the model distortion map are shown in Figure 4. These vectors have been obtained by applying the distortion correction model to a regularly spaced grid of points and fitting and subtracting a 6-parameter linear transformation to each chip. The 
characteristic circular pattern is seen in other H2RG chips, including for the ShaneAO SHARCS detector (Figure 5), and may be a reflection of anisotropic pixel centers or sizes.

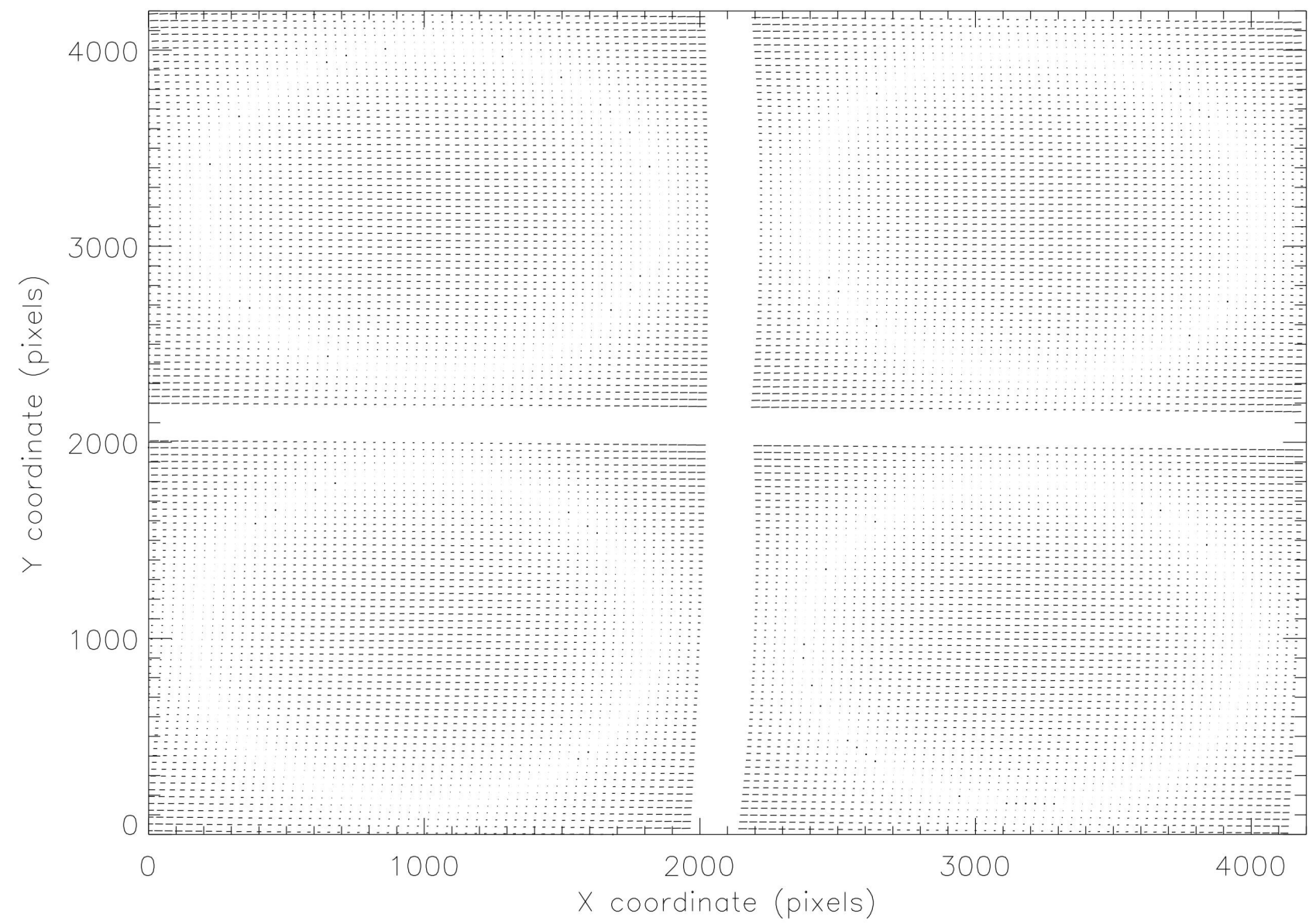

Figure 4. Nonlinear components of GSAOI distortion map.

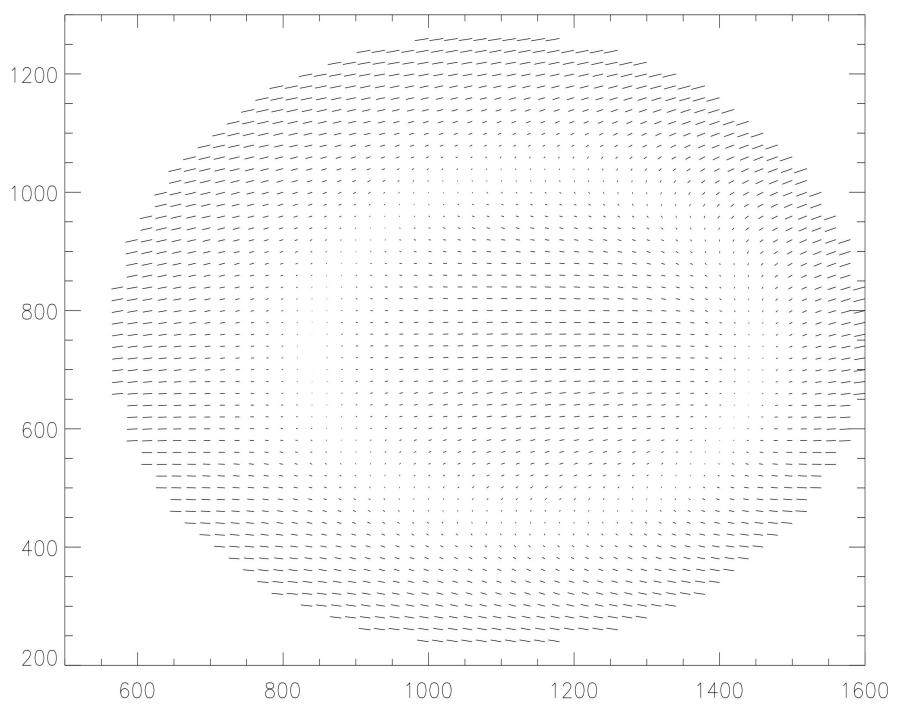

Figure 5. Nonlinear components of ShaneAO SHARCS distortion map.

Proc. of SPIE Vol. 9909 99095T-5 


\subsection{GeMS Luhman 16 AB Astrometry}

The GSAOI images of Luhman $16 \mathrm{AB}$ were analyzed by first running Starfinder ${ }^{16}$ on individual frames. A 16x16 pixel image of Luhman A is used as the reference PSF for cross-correlation with other stars in the field. The correlation between the Luhman $16 \mathrm{~A}$ and Luhman B PSFs is consistently above 0.99 and the median value over all frames is 0.994 . We establish a set of common stars in all frames using a procedure similar to that described above for the NGC1851 analysis. If Starfinder splits a single star into multiple detections in single frame, those measurements are excluded (but other frames with a single detection are retained for that star).

The frames are distortion corrected using the distortion map measured from NGC1851 images, as described in the section above. Following distortion correction, the stellar positions for all epochs are transformed to the same astrometric frame with 6-parameter linear transformations measured from $\sim 14$ background stars. The errors on the Luhman 16 AB separation are estimated by computing the standard deviation of the separation for a given epoch and dividing by the root of the number of observations in the epoch. The errors on the mean vary between 0.16 and 0.28 milliarcseconds for each epoch.

\subsection{ESO R-band Astrometry}

We make use of a scanned plate image of Luhman 16 AB taken by the ESO 1-meter Schmidt Telescope at La Silla Observatory on March 1, 1984, first identified by Mamajek. ${ }^{10}$ SUPERCOSMOS ${ }^{17}$ obtained a scan of ESO red plate $R 5562$ with a plate scale of 0.675 " on May 15 , 2001. The filter used for the original photographic plate was the RG630 filter and the plate used IIIaF emulsion. The exposure time was 120 seconds.

Luhman $16 \mathrm{AB}$ is fully resolved in the 1984 epoch. We modify the IDL code mpfit2dpeak to fit two Lorentzian PSFs to the 1984 data, using Lorentzian widths fit to isolated, unsaturated background stars on the photographic plate.

\subsection{MCMC Orbital Fit}

We use the Python affine-invariant parallel-tempered ensemble sampler PTsampler function with the emcee package ${ }^{18}$ to perform orbital fits to the 7 epochs of relative astrometry from GeMS and the ESO R-band photographic plate. We adopted uniform priors in total dynamical mass, semi-major axis, eccentricity, longitude of ascending node, argument of periastron, and time of periastron passage. We assume randomly distributed viewing angles by adopting a uniform prior in inclination. We used 1000 walkers and 10000 steps, and discarded the first 20\% of steps as the burn-in for each walker. We compute posterior probability distributions of each of the six model parameters by marginalizing over the other five.

A representative orbit fit and residuals for the GeMS and ESO plate data is shown in Figure 6. The ESO plate data from 1984 provides a good constraint on period, but the limited orbital coverage of 
the GeMS data produces a degeneracy between total system mass, eccentricity, and inclination. Incorporation of other archival data in the 1995-2005 epoch, which is approximately half a period into the past, will greatly reduce this degeneracy and allow the total mass to be determined (Garcia et al. 2016, in prep.).

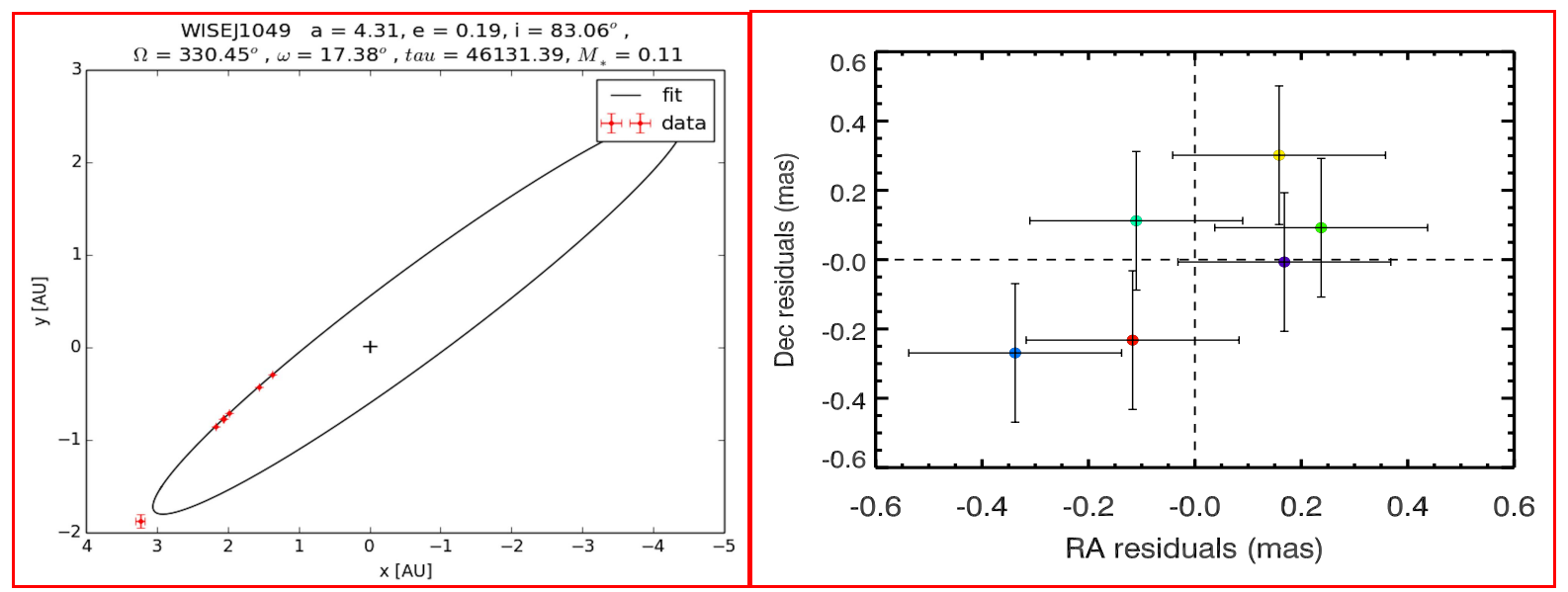

Figure 6. Left: Representative orbital fit to GeMS and ESO plate data. The plate data from 1984 is shown in the bottom left corner of the image. Right: Residuals of the GeMS data in milliarcseconds (ESO plate data not shown). Orbital fits to 2 years of GeMS monitoring yield total system masses between 0.06 and 0.11 solar masses and residuals of 0.22 milliarcseconds. Due to the incomplete sampling of the orbit with the existing data and the highly inclined orbit, there is a degeneracy between total system mass, inclination, and eccentricity. This degeneracy will eventually be broken with further monitoring in 2016 and incorporation of further archival data.

\subsection{Limits on the Presence of Low-Mass Companions}

Significant correlated residuals in previous astrometric attempts to constrain the total mass of the system suggested the presence of a low-mass companion orbiting one of the components. ${ }^{19}$ Here, we place limits on the presence of a low-mass companion orbiting either component of Luhman $16 \mathrm{AB}$ through Monte Carlo simulation of potential planetary orbits and re-fitting of the resulting relative orbit data.

We simulate potential orbiting planets by creating 10000 circular orbits in each of $10 \times 10$ bins of companion mass and period. The companion masses tested ranged from 2 earth masses to 5000 earth masses. The periods tested ranged from 50 days to 10000 day periods. Semimajor axis was computed explicitly from the specified period and primary star mass using Kepler's law. The other orbital elements were randomized about angle (inclination, longitude of ascending node, and argument of periapsis). The orbits were simulated for each of two primary star cases -25 Jupiter masses for the $\mathrm{T}$ dwarf, and 35 Jupiter masses for the $\mathrm{L}$ dwarf. The resulting astrometric perturbation on the stellar separation was sampled at the six GeMS epoch times.

For each simulated planet orbit, the Luhman $16 \mathrm{AB}$ mutual orbit was refit to the perturbed data and the residuals characterized. If the residuals were a factor of two greater than the individual epoch error bars, the presence of a perturbing planet was considered "detected" (although without constraint on planetary orbital elements). If the residuals were less than a factor of two times the 
epoch error bars, that planet was considered undetected. The resulting probability of "detection" is shown in Figure 7 as a function of planet mass and period. Nearly $100 \%$ of all Saturn mass planets in orbits with periods between 300 days and 1000 days are ruled out for both $\mathrm{L}$ and $\mathrm{T}$ dwarf components.
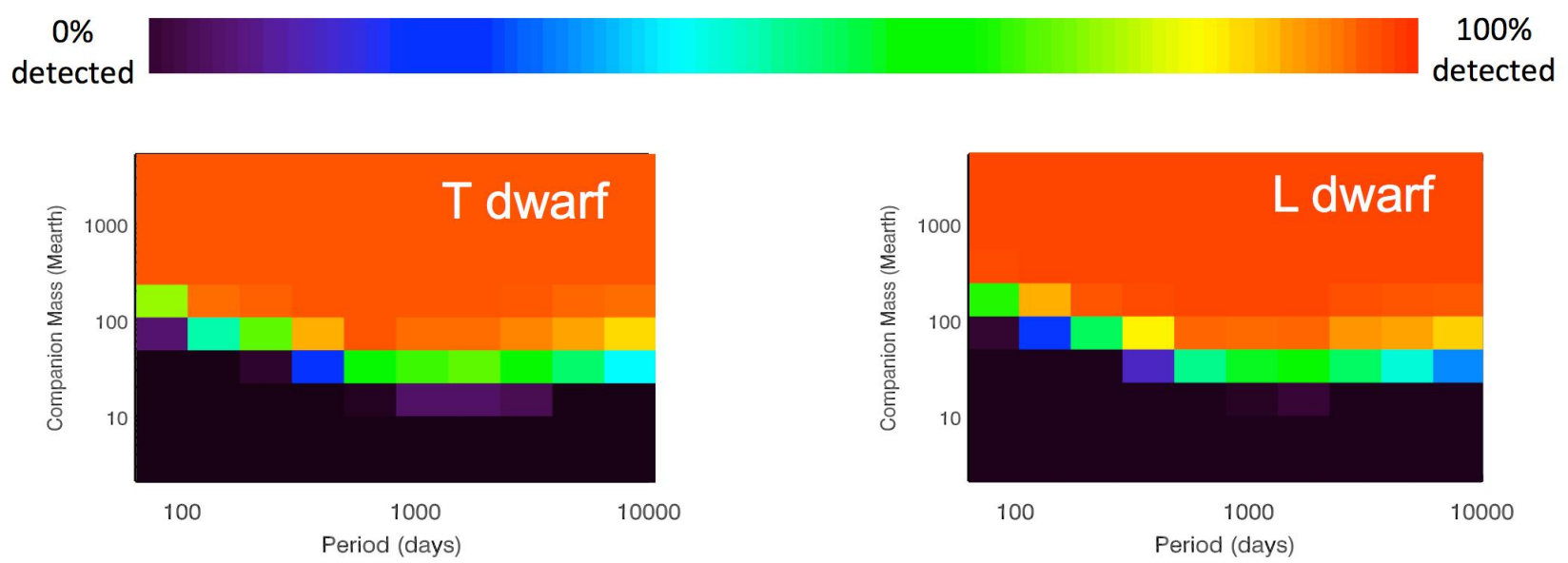

Figure 7. The lack of significant deviations from regular orbital motion places tight constraints on the presence of exoplanets orbiting either dwarf in Luhman 16 AB. Nearly $100 \%$ of Saturn mass planets in all orbits with periods between 300 and 1000 days are ruled out.

\section{CONCLUSION}

In this paper, we constrained the mutual orbit of Luhman 16AB by combining highly precise astrometric data from the GeMS MCAO system with a quantitative analysis of a 1984 photographic plate of the system. A MCMC fit to both data sets with uniform priors in all orbital elements results in total system masses between 60 and 110 Jupiter masses. The highly inclined orbital configuration of Luhman $16 \mathrm{AB}$ results in a degeneracy between total system mass, eccentricity, and inclination. Further monitoring in 2016-2017 as the system approaches its minimum projected separation will significantly reduce this degeneracy. The GeMS residuals indicate that narrow-field astrometric precisions of 0.2 mas are possible with MCAO imaging systems over timescales of multiple years.

\section{ACKNOWLEDGEMENTS}

We acknowledge all members of the Shane AO commissioning team for assistance in obtaining M92 images. Based on observations obtained at the Gemini Observatory, which is operated by the Association of Universities for Research in Astronomy, Inc., under a cooperative agreement with the NSF on behalf of the Gemini partnership: the National Science Foundation (United States), the National Research Council (Canada), CONICYT (Chile), the Australian Research Council (Australia), Ministerio da Ciencia, Tecnologia e Inovacao (Brazil) and Ministerio de Ciencia, Tecnologia e Innovacion Productiva (Argentina). Data are acquired through the Gemini Science Archive and processed using the Gemini IRAF package. This research is based on the data obtained from the ESO Science Archive Facility under programme IDs 291.C-5004 and 593.C-0314. This 
research has made use of data obtained from the SuperCOSMOS Science Archive, prepared and hosted by the Wide Field Astronomy Unit, Institute for Astronomy, University of Edinburgh, which is funded by the UK Science and Technology Facilities Council. This work was partially funded by NASA/NEXSS NNX15AD95G. This research has made use of the VizieR catalogue access tool, CDS, Strasbourg, France. This work performed under the auspices of the U.S. Department of Energy by Lawrence Livermore National Laboratory under Contract DE-AC52-07NA27344 with document release number LLNL-PROC-701398.

\section{REFERENCES}

[1] Marois, C., et al. "Direct Imaging of Multiple Planets Orbiting the Star HR 8799," Science, 322, 1348 (2008).

[2] Lafrenière, D., et al. "Direct Imaging and Spectroscopy of a Planetary-Mass Candidate Companion to a Young Solar Analog," ApJ, 689, 153 (2008).

[3] Kalas, P., et al. “Optical Images of an Exosolar Planet 25 Light-Years from Earth,” Science, 322, 1345 (2008).

[4] Lagrange, A.-M., et al. "A probable giant planet imaged in the $\beta$ Pictoris disk. VLT/NaCo deep L'-band imaging," A\&A, 493, 21 (2009).

[5] Macintosh, B., et al. "Discovery and spectroscopy of the young jovian planet 51 Eri b with the Gemini Planet Imager," Science, 350, 64 (2015).

[6] Shao, M., et al. "The Synergy of Direct Imaging and Astrometry for Orbit Determination of Exo-Earths," ApJ, 720, 357 (2010).

[7] Guyon, O., et al. "High-precision Astrometry with a Diffractive Pupil Telescope," ApJS, 200, 11 (2012)

[8] Makarov, V., et al. "Starspot Jitter in Photometry, Astrometry, and Radial Velocity Measurements," ApJL, 707, 73 (2009).

[9] Luhman, K. "Discovery of a Binary Brown Dwarf at 2 pc from the Sun,” ApJL, 767, 1 (2013).

[10] Mamajek, E., et al. "On the Nearby Binary Brown Dwarf WISE J104915.57-531906.1 (Luhman 16)," arXiv:1303.5345 (2013).

[11] Sahlmann, J. \& Lazorenko, P.F. "Mass ratio of the 2 pc binary brown dwarf LUH 16 and limits on planetary companions from astrometry," MNRAS, 453, 103 (2015).

[12] Mcgregor, P., et al. "Gemini South Adaptive Optics Imager (GSAOI)," proc. SPIE, 5492, 1033 (2004).

[13] Carrasco, E.R., et al. "Results from the commissioning of the Gemini South Adaptive Optics Imager (GSAOI) at Gemini South Observatory," proc. SPIE, 8447, 0N (2012).

[14]Ford, H., et al. “Advanced camera for the Hubble Space Telescope,” proc. SPIE, 3356, 234 (1998).

[15] Tody, D. “The IRAF Data Reduction and Analysis System," proc. SPIE, 627, 733 (1986).

[16] Diolaiti, E., et al. "StarFinder: an IDL GUI-based code to analyze crowded fields with isoplanatic correcting PSF fitting”, Proc. SPIE, 4007, 879 (2000).

[17] Hambly, N., et al. “The SuperCOSMOS Sky Survey - I. Introduction and description,” MNRAS, 326, 1279 (2001).

[18] Foreman-Mackey, D., et al. “emcee: The MCMC Hammer,” PASP, 125, 306.

[19] Boffin, H., et al. "Possible astrometric discovery of a substellar companion to the closest binary brown dwarf system WISE J104915.57-531906.1,” A\&A, 561, 4. 